\title{
105 \\ Image Browsing: a Space-Time Trade-off
}

\author{
Kevin Lam and Robert Spence \\ Department of Electrical \& Electronic Engineering \\ Imperial College, London, England SW7 2BT \\ +441715946259 \\ r.spence@ic.ac.uk
}

\begin{abstract}
Browsing is defined as the assessment of content, and is different from searching. Whereas the browsing of text can be tedious and difficult, the browsing of images can often proceed more easily and at a much faster rate. For example, while images may well occupy more space than the associated descriptive text, their adequate appearance need only be brief. For this reason, the space that would be required to fully and continuously display a number of images can be traded for a much smaller space if those images are capable of being riffled, each image being displayed only momentarily. The space-time trade-off is illustrated by the example of a video-store whose contents can be browsed.
\end{abstract}

\section{KEYWORDS}

Browsing, Visualization

\section{BROWSING}

Browsing - a term often informally used to describe a variety of activities - is here defined solely as the assessment of content. A new book, for example, may be picked up, and its pages flicked through in a matter of seconds, to gain some awareness of structure, makeup, features and style - in general, an awareness of content. Browsing is also well illustrated by a diner viewing the menu in a new restaurant, or the person having a first glance at The Times newspaper.

Browsing is not the same as searching, the latter activity being characterised by a specific goal. While searching often follows browsing or, in some cases, is essentially concurrent with browsing, it is nevertheless important that interaction design should specifically address the needs of browsing as a distinct activity.
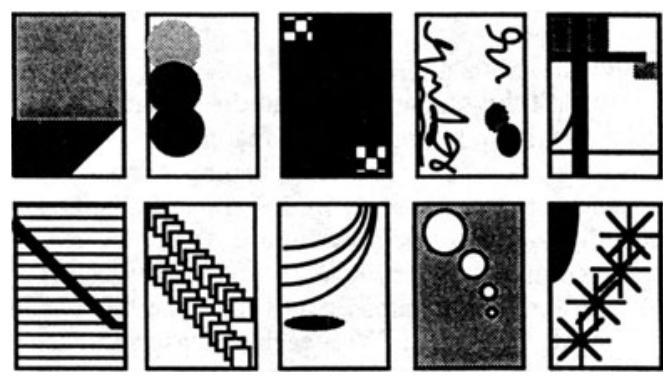

Figure 1 Images in full view

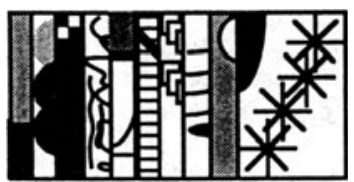

Figure 2 Images partially overlapped to conserve space

\section{A SPACE-TIME TRADE-OFF}

It can be tedious and time-consuming, for example, to browse a textual list of paintings in a collection. By comparison, even a brief glance at the paintings themselves may well be sufficient to gain some insight into the nature of that collection. However, if images are displayed in full (Figure 1), an immediate disadvantage with a display screen of finite size is a severe limit on the number of images that can simultaneously be displayed, at adequate resolution, for perception, retention and later recognition. Overlapping (Figure 2) of the images goes some way towards ameliorating this space limitation, but at the expense of only a partial presentation of each image. 


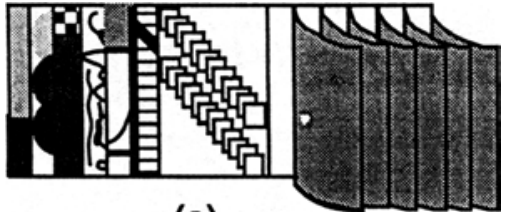

(a)

(b)

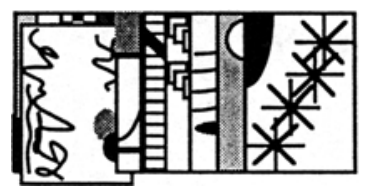

Figure 3 Each image is fully though momentarily displayed

A remarkably simple solution to the space limitation is to exploit the human being's ability [4] to perceive, retain and later recognise the features of an image following its relatively brief display. Thus, if the images are now displayed in full only momentarily, either by a riffling action (Figure $3 \mathrm{a}$ ) or by a brief though complete display of each image in turn (Figure $3 \mathrm{~b}$ ), the 'browsing capacity' of the display can be significantly increased. With each of these approaches, space has been traded against elapsed time.

\section{A VIDEO STORE}

The 'space-time trade-off' approach to image browsing has been illustrated in the context of a video store in which a customer might first browse to see what videos are available, later gradually moving towards a search activity to select a video for viewing. Though our primary concern here is with browsing, the interface design fully recognises the need, additionally, to support effective search.

The appearance of the video store is shown in Figure 4. There are three rows ('shelves') of video posters. All except those in the centre region are overlapped to a considerable degree, though complete overlap is avoided because of the need to show context and approximate extent and to provide spatial reference. The bifocal concept [3] supports the concurrent display of 'focus and context', and is combined with the '3D' Perspective Wall [2] presentation technique thus, during and after browsing, some posters representing individual videos can be examined in more detail in the central region.

Browsing to assess the structure and content of the store is achieved by simple movement of the cursor over the overlapped collections of posters at each side. As the cursor moves over a poster, it immediately protrudes allowing full view. Typically, the entire row might be browsed in three or four seconds.

Other features are conventional. For example, as in the bifocal display [3], the entire 'shelving' can be moved to and fro to bring particular categories of video ('musical', 'education') into the central region, and to allow a poster-based menu to be used for additional browsing or search. The example is, of course, neither the only nor the first example of the appropriateness, for browsing, of the momentary display of an image. The Video Streamer [1], for example, is a superb and earlier example. The purpose of the video is to demonstrate an essentially dynamic technique by video.

\section{CONCLUSION}

Given that browsing is the assessment of content, the browsing of images can be achieved efficiently in limited space by the activity of riffling, with each image only displayed for a fraction of a second.

\section{REFERENCES}

1 Elliot, E. and Davenport, G. Video Streamer, ACM, CHI'94 Companion Proceedings, pp.65-66 (1994)

2. Mackinlay, J .D., Robertson, G.G. and Card, S.K., The Perspective Wall: detail and context smoothly integrated, ACM, Proc. CHI'91, pp.173-179 (1991)

3. Spence, R. and Apperley, M.D. Data-base navigation: an office environment for the professional, Behaviour and Information Technology, 1, 1, 43-54 (1982).

4. Standing, L. (1973) Learning 10,000 pictures, Quarterly Journal of Experimental Psychology, 25, 207222 (1973)

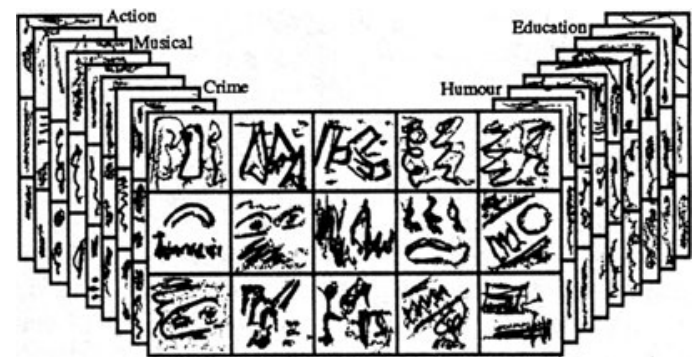

Figure 4 Sketch of the implemented video store 